\title{
Combining forecasts in short term load forecasting: Empirical analysis and identification of robust forecaster
}

\author{
YOGESH K BICHPURIYA ${ }^{1, *}$, S A SOMAN ${ }^{1}$ and A SUBRAMANYAM ${ }^{2}$ \\ ${ }^{1}$ Department of Electrical Engineering, Indian Institute of Technology Bombay, Mumbai 400076, India \\ ${ }^{2}$ Department of Mathematics, Indian Institute of Technology Bombay, Mumbai 400076, India \\ e-mail: bichpuriya@iitb.ac.in
}

MS received 19 January 2015; revised 13 January 2016; accepted 19 April 2016

\begin{abstract}
We present an empirical analysis to show that combination of short term load forecasts leads to better accuracy. We also discuss other aspects of combination, i.e., distribution of weights, effect of variation in the historical window and distribution of forecast errors. The distribution of forecast errors is analyzed in order to get a robust forecast. We define a robust forecaster as one which has consistency in forecast accuracy, lesser shocks (outliers) and lower standard deviation in the distribution of forecast errors. We propose a composite ranking (CRank) scheme based on a composite score which considers three performance measures-standard deviation, kurtosis of distribution of forecast errors and accuracy of forecasts. The CRank helps in identification of a robust forecasts given a choice of individual and combined forecaster. The empirical analysis has been done with the real life data sets of two distribution companies in India.
\end{abstract}

Keywords. Combination; composite score; robustness; short term load forecasting.

\section{Introduction}

Load forecasting is an important problem for optimal planning and operation of the electricity markets. Short term load forecasting (STLF) methods have been discussed for more than five decades and still have found interests of many researchers and industry analysts. A variety of methods have been referred in a recent survey paper Hahn et al [1] to deal with forecasting of electrical load in short term. The performance of these methods varies from one data set to another, e.g., STLF using time series approaches e.g., integrated auto-regressive moving average (ARIMA) works well for an electricity distribution company (discom), whereas, an artificial neural network (ANN) model gives better results for another. Even on a common data set, different models usually produce different forecasts. These forecasts differs due to the process which involves selection of explanatory variables and model, collection of data, model validation and forecasting. Forecasters that differ at any step of the process will lead to different forecasts.

A standard practice followed in forecasting is to first experiment with multiple models and then select one with the satisfactory performance [in terms of mean absolute percentage error (MAPE), sum of squared error (SSE), etc.] on a data set under consideration. We usually discard the methods which have relatively poor performance. However, the discarded forecasts may have some independent

*For correspondence information. In some cases, the information may even help in reducing the forecast error. This motivates the researchers in the field of forecasting to think upon combination of multiple forecasts.

Combination of forecasts has been an important area of research after the seminal paper by Bates and Granger [2]. Various aspects of combining forecasts have been studied. Wallis [3] provided further progress in titled as Combination of forecasts-forty years later. Bichpuriya et al [4] discussed combination of forecasting in the context of STLF. These combination methods are being applied to many other areas of forecasting e.g., price forecasting Borgignon et al [5], tourism forecasting Shen et al [6], etc.

In this paper, we discuss combination of STLF generated using three different forecasting models viz. similar day approach (SDA), ANN and ARIMA. All the three models use same information set to forecast day-ahead load profile. The idea is to get an overview of the state of the art in generic forecasting literature and then to compare and contrast with the STLF problem. We present an empirical analysis of combination approach in the context of STLF.

We define a robust forecaster as one which has consistency in forecast accuracy, lesser shocks (outliers) and lower standard deviation in the forecast error distribution. We propose a strategy to develop a robust forecaster by using combination approaches. We also propose a composite score (cs) formula which considers three performance measures-standard deviation, kurtosis of forecast error distribution and MAPE. The composite score for each 
forecaster has been calculated after analyzing their out-ofsample performance for three months. A composite rank (CRank) has been assigned to each forecaster. A forecaster with the highest rank is identified as a robust forecaster. The proposed framework validates the robustness of the identified forecaster.

The paper is organized as follows. Section 2 provides a review of the approaches for combining forecasts for generic forecasting as well as STLF in specific. In section 3, we discuss robustness of a forecaster and propose a ranking scheme based on composite score for the robustness of a forecaster. Section 4 presents extensive empirical investigation of combination approaches with real life data sets of two discoms. Section 5 concludes the paper.

\section{Review of approaches for combining forecasts}

Definition 1 Let $\mathbf{F}_{T}=\left[f_{1, T}, f_{2, T}, \ldots, f_{N_{f}, T}\right]$ be a set of $N_{f}$ load forecasts at time $T$ by different methods or from different information sets. Then the linear combination of forecasts can be represented as

$$
f_{c, T}=\sum_{i=1}^{N_{f}} w_{i, T} f_{i, T}=\mathbf{F}_{T} \mathbf{w}_{T}
$$

where $\mathbf{w}_{T}=\left[w_{1, T}, w_{2, T}, \ldots, w_{N_{f}, T}\right]^{\prime}$ be an $N_{f} \times 1$ column vector of weights assigned to the individual forecasts at time $T$.

While some of the combination approaches permit unrestricted weights, others put additional conditions on weights to be non-negative and that their sum to be unity i.e.,

$$
\begin{gathered}
\mathbf{u}^{\prime} \mathbf{w}_{T}=1 \\
\mathbf{w}_{T} \geq 0
\end{gathered}
$$

where $\mathbf{u}=[1,1, \ldots, 1]^{\prime}$ be a unity vector of $N_{f} \times 1$. Both of these constraints put together implies a convex combination of forecasts. Some methods pose a condition on candidate forecasts to be unbiased. A biased forecast is one, which overestimates or underestimates most of the time i.e., the forecast error does not have a zero mean. Convex combination of unbiased forecast with a biased one would lead to a biased forecast. However, even if all candidate forecasts are unbiased, it would not mean that the combined forecasts would necessarily be unbiased. We will discuss implications of using the additional constraints while reviewing the various combination methods.

Now, consider a set of candidate forecasts, $\mathbf{F}=\left[\mathbf{f}_{1}, \ldots, \mathbf{f}_{N_{f}}\right]$, an $N \times N_{f}$ matrix of forecast values using $N_{f}$ forecasting methods, where $N$ is the number of historical observations considered to obtain the weights. Let $\mathbf{y}$ be an
$N \times 1$ vector of historical realizations of random variable i.e., load. The forecast error at time $t$ can be calculated as

$$
e_{i, t}=y_{t}-f_{i, t} \quad \forall i=1,2, \ldots, N_{f} .
$$

For historical data window of size $N$, e will be of size $N \times N_{f}$ where rows correspond to realizations of $\mathbf{x}$ and columns represent forecasting methods. Now, the covariance matrix can be calculated as

$$
\mathbf{V}=\mathbf{E}\left[\mathbf{e}^{\prime} \mathbf{e}\right]
$$

Variance of error in combined forecasts can be given by

$$
\sigma_{c}^{2}=\mathbf{w}^{\prime} \mathbf{V w}
$$

In practice, the covariance matrix is not known. A sample covariance matrix from the historical sample can be used but it could be sensitive to change in the data size. Use the sum of squared error (sse) for each forecaster, assuming the forecasts' error have zero mean, is one of the alternatives to variance.

$$
\operatorname{sse}_{i}=\sum_{t=T-N}^{T-1} e_{i, t}^{2} .
$$

Many combination methods have been developed and studied for various objectives. In some methods, optimal weights depend on the objective function that is being minimized. We, now, discuss commonly referred combination methods listed in table 1 . These methods are discussed in the areas like economic forecasts and airline passenger forecasts.

\subsection{Combination of short term load forecasts}

Even though the combination approaches have been applied widely in many other areas, only few attempts are made in the field of STLF. Kiartzis et al [10] applied Bayesian combination approach for combining ANN and linear regression predictors. Barghinia et al [11] discussed combination of STLF from three models viz. neurofuzzy, Bayesian and SDA. They applied least square method to combine the forecasts. Fan et al [12] discussed combination of weather forecasts from different sources using aggregated forecast from exponential reweighting (AFTER). The combined weather forecast is used in ANN for STLF. Multiple sets of ANN training data are generated using bootstrap sampling and then all the forecasts are combined with equal weights. For combining STLF, Bichpuriya et al [4] proposed following four approaches.

- Median of forecasts (Median)

- Combining with weights proportional to probability of success (PoS)

- Combining with weights calculated by variance minimization (MinVarC) 
Table 1. Summary of combination approaches.

\begin{tabular}{|c|c|c|}
\hline Combination approach & Description & Remark \\
\hline Arithmetic mean (AVG) & $w_{i}=1 / N_{f}$ & $\begin{array}{l}\text { Simple method, used as a reference for comparing other } \\
\text { combination approaches, weights are stable as they do not } \\
\text { depend on historical performance of the forecasts }\end{array}$ \\
\hline $\begin{array}{l}\text { Minimization of variance of error in } \\
\text { combined forecast (BG) }\end{array}$ & $w_{i}=\hat{\sigma}_{i}^{-2} / \sum_{i=1}^{N_{f}} \hat{\sigma}_{i}^{-2}$ & $\begin{array}{c}\text { Bates and Granger [2], assuming zero correlation between the two } \\
\text { forecasts' error vector }\end{array}$ \\
\hline Minimum sum of squared error (SSE) & $w_{i}=s s e_{i}^{-1} / \sum_{i=1}^{N_{f}} s s e_{i}^{-1}$ & $\begin{array}{l}\text { Bates and Granger [2], assuming the forecasts' error have zero } \\
\text { mean, weights are non-negative and sum to unity }\end{array}$ \\
\hline Minimization of variance (MinVar) & $\mathbf{w}_{T}=\frac{V^{-1} \mathbf{u}}{\mathbf{u}^{\prime} V^{-1} \mathbf{u}}$ & $\begin{array}{c}\text { Newbold and Granger [7], generalization of minimization of } \\
\text { variance method, considered full covariance matrix and } \\
\text { weights can be negative }\end{array}$ \\
\hline $\begin{array}{l}\text { Unrestricted least square estimation } \\
\text { methods (LSE-1) }\end{array}$ & $\min _{\mathbf{w}}\|(\mathbf{x}-\mathbf{F w})\|_{2}$ & $\begin{array}{c}\text { Granger and Ramanathan [8], does not guarantee that weights } \\
\text { sum to unity, it may not lead to an unbiased forecast even if the } \\
\text { individual forecasts are unbiased }\end{array}$ \\
\hline Constrained LSE (LSE-2) & $\min _{\mathbf{w}}\|(\mathbf{x}-\mathbf{F w})\|_{2}, \mathbf{u}^{\prime} \mathbf{w}=1$ & $\begin{array}{c}\text { Granger and Ramanathan [8], the constraint may help to get the } \\
\text { unbiased combined forecast if the candidate forecasts are } \\
\text { unbiased, weights can be negative }\end{array}$ \\
\hline $\begin{array}{l}\text { Adding a constant in linear regression } \\
\text { (LSE-3) }\end{array}$ & $\min _{w_{0}, \mathbf{w}}\left\|\left(x \mathbf{x}-w_{0} \mathbf{u}-\mathbf{F w}\right)\right\|_{2}$ & $\begin{array}{c}\text { Granger and Ramanathan [8], Adding a constant term corrects the } \\
\text { bias for all the candidate forecasts and leads to an unbiased } \\
\text { combined forecast, the value of objective function is less than } \\
\text { the unrestricted LSE problem }\end{array}$ \\
\hline $\begin{array}{l}\text { Minimizing Absolute Percentage Error } \\
\text { (APE) for each time interval } \\
\text { (MinAPEi) }\end{array}$ & $\min _{\mathbf{w}}\left|\frac{y_{j}-\mathbf{F}_{j} \mathbf{w}}{y_{j}}\right|$ & $\begin{array}{c}\text { Lam et al [9], the optimization problem can be converted to a } \\
\text { Linear Programming (LP) problem }\end{array}$ \\
\hline $\begin{array}{l}\text { Minimizing maximum APE } \\
\text { (MinMaxAPEi) }\end{array}$ & $\min _{\mathbf{w}}\left(\max \left|\frac{y_{j}-\mathbf{F}_{j} \mathbf{w}}{y_{j}}\right|\right)$ & $\begin{array}{c}\text { Lam et al [9], the optimization problem can be converted to a } \\
\text { Linear Programming (LP) problem }\end{array}$ \\
\hline
\end{tabular}

$$
\min _{\mathbf{w}} \frac{1}{2} \mathbf{w}^{\prime} \mathbf{V} \mathbf{w} \quad \text { s.t. } \quad \mathbf{u}^{\prime} \mathbf{w}=1, w_{i} \geq 0 .
$$

- Combining with weights calculated from eigenvector of covariance matrix of forecast errors (Eigenvec)

$$
w_{i}=\frac{\left|p_{i N_{f}}\right|}{\sum_{i=1}^{N_{f}}\left|p_{i N_{f}}\right|}
$$

where $p_{i}$ is the normalized eigenvector of the covariance matrix $V$.

\section{Robustness of forecaster}

We have discussed many combination approaches in section 2. Except averaging techniques, all other combination approaches derive the weights using historical performance of the individual forecasts. The weights may not be optimal for out-of-sample testing and hence, the combination methods may not perform as expected. For instance, minimization of variance methods depends on the variances of forecast errors of individual methods and correlation between the two forecast errors. We estimate the variance from the historical samples. Instability in sample variance at different time periods leads to instability in the weights derived using minimization of variance. The instability in weights may result in a variation of performance of combination approaches based on the minimization of variance.

The combination approaches obtain weights using different objectives e.g., minimum variance, minimum sum of squared error, etc. Comparison of the all combination approaches using a single metric may be spurious. Even, evaluating a particular combination approach based on an accuracy measure like SSE or MAPE would result in ignoring the statistics of distribution of forecast errors. The distribution of errors may help in estimating the risk and uncertainty associated with the forecasters.

After doing empirical analysis of various combination approach in the context of short term load forecasting, we suggest a strategy, as shown in figure 1, for selecting a robust forecaster (combination or individual). We define a robust forecaster as one, which has consistent performance in terms of either MAPE or SSE and distribution of forecast error does not have longer tail (i.e., outliers).

We also define a composite measure for robustness called CRank as follows. Let $\mathcal{F}$ be a given set of $N_{\mathcal{F}}$ individual and combination forecasters. Robustness of a forecaster $i \in \mathcal{F}$ is measured by statistics of error distribution e.g., standard deviation $\left(\sigma_{i}\right)$ and kurtosis $\left(\beta_{2_{i}}\right)$, and measure of accuracy e.g., MAPE, $\left(e_{i}\right)$. Let $\hat{\sigma}, \hat{\beta_{2}}$ and $\hat{e}$ be the sample average of $\boldsymbol{\sigma}, \boldsymbol{\beta}_{\mathbf{2}}$ and $\mathbf{e}$ respectively e.g., 


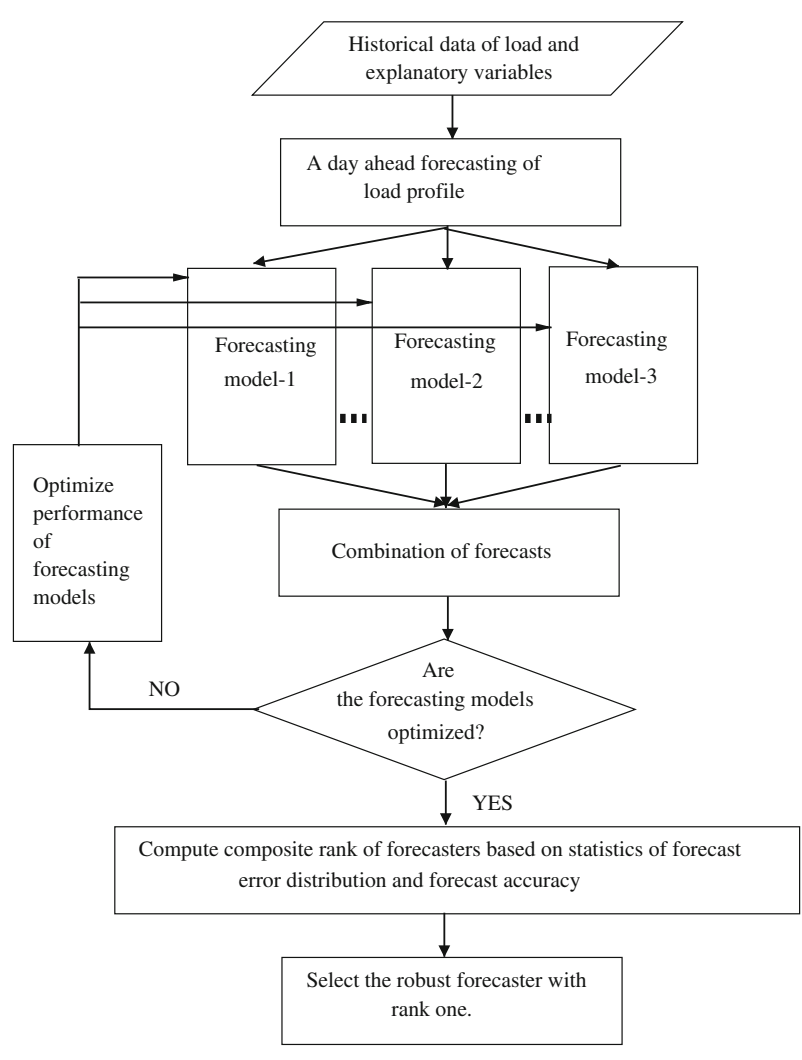

Figure 1. Block diagram for developing a robust forecaster.

$\hat{\sigma}=\frac{1}{N_{\mathcal{F}}} \sum_{i=1}^{N_{\mathcal{F}}} \sigma_{i}$. Use of kurtosis in the context of outliers and robustness is discussed in DeCarlo [13]. A composite score $\left(c s_{i}\right)$ for a forecaster $i$ can be calculated as

$$
c s_{i}=w_{1}\left(\sigma_{i}-\hat{\sigma}\right)^{2 n+1}+w_{2}\left(\beta_{2_{i}}-\hat{\beta_{2}}\right)^{2 n+1}+w_{3}\left(e_{i}-\hat{e}\right)^{2 n+1} .
$$

Weights, $\left(w_{1}, w_{2}, w_{3}\right)$, are non-negative and subjective but sum to one. A decision maker can assign weights in proportion to the preferences over one measure to other. In power index $2 n+1, n$ could be substituted with 0,1 or some higher integer. Hence, bias towards lower or best values is encouraged by the power index of the odd number. If a forecaster is better than average forecaster in all the three respects, then each of the terms will be negative and the overall score will also be negative. Thus, smaller values of composite score indicate robust forecaster. In general, lower values are better and higher being worse. For the rank one forecaster, all the three terms in right hand side of Eq. (10) should have negative values which implies that the top ranked forecaster is better than the average of all the individual performance measures. If this is not a case then it indicates that the forecasters can be optimized further for better accuracy or we may require to consider a larger set of forecasters.

\section{Case study}

We evaluate the performance of combination approaches on the load data of two discoms in the city of Mumbai. These discoms differ in consumer mix and volume of supply. Discom-1 has a mix of industrial, railways, residential and commercial consumers. The residential consumers' load has less proportion in load profile of the discom-1 as compared to other consumers categories. The load of discom-1 on a typical day varies from 150 to 380 MW. Discom-2 supplies mostly to residential and commercial consumers. The load of discom- 2 on a typical day varies from 200 to $800 \mathrm{MW}$.

In India, the discoms have to submit their next day load profile, which has 96 points at the interval of $15 \mathrm{~min}$, to their state load despatch centres. Any deviation from the submitted schedule in real time may result in financial loss to the Discom. The discoms, therefore, would like to predict the next day load profile with good accuracy. For STLF, we apply three forecasting models viz. SDA, ANN and ARIMA.

Table 2. List of combination approaches for comparison.

\begin{tabular}{lc}
\hline Category & Combination approach \\
\hline Averaging & Arithmetic mean (AVG) \\
Outperformance & Median of forecasts (MED) \\
Minimization of variance & Probability of success (PoS) \\
& Bates and Granger method (BG) \\
& Minimum sum of squared error (SSE) \\
& Minimization of variance (MinVar) \\
& Minimization of variance with constraint on weights (MinVarC) \\
Least square estimation & Eigenvector of covariance matrix (Eigenvec) \\
& Unconstrained combination (LSE-1) \\
Minimization of APE & Sum of weights to be unity (LSE-2) \\
& Adding a constant in linear regression (LSE-3) \\
& Minimizing APE for each time interval (MinAPEi) \\
& Minimizing maximum APE (MinMaxAPEi)
\end{tabular}


Table 3. Comparison of MAPE and SSE of candidate forecasts and their combinations for discom-1 from August 2013 to October 2013.

\begin{tabular}{|c|c|c|c|c|c|c|c|}
\hline \multicolumn{2}{|l|}{ Forecasting methods } & \multicolumn{3}{|c|}{ MAPE (\%) } & \multicolumn{3}{|c|}{$\operatorname{SSE}\left(\times 10^{4}\right)$} \\
\hline Category & Model & August & September & October & August & September & October \\
\hline \multirow{3}{*}{ Candidate forecasts } & ANN & 7.28 & 7.54 & 7.59 & 11.69 & 14.83 & 16.83 \\
\hline & SDA & 8.92 & 8.69 & 8.66 & 16.61 & 16.14 & 16.30 \\
\hline & ARIMA & 6.82 & 6.88 & 5.27 & 12.76 & 14.25 & 9.59 \\
\hline \multirow[t]{2}{*}{ Averaging } & Average & 5.34 & 6.19 & 5.66 & 6.56 & 9.96 & 8.54 \\
\hline & Median & 5.36 & 6.35 & 5.70 & 7.05 & 10.79 & 8.86 \\
\hline Outperformance & $\mathrm{PoS}$ & 4.26 & 5.17 & 4.35 & 4.74 & 8.13 & 6.39 \\
\hline \multirow[t]{5}{*}{ Minimization of variance } & BG & 5.75 & 6.33 & 5.84 & 8.43 & 10.84 & 9.10 \\
\hline & MinVar & 7.65 & 7.16 & 6.70 & 14.65 & 14.12 & 13.94 \\
\hline & SSE & 4.46 & 5.21 & 4.37 & 5.26 & 8.25 & 6.19 \\
\hline & MinVarC & 6.35 & 6.34 & 5.88 & 11.10 & 11.83 & 10.78 \\
\hline & Eigenvec & 6.18 & 6.66 & 6.05 & 9.37 & 11.68 & 9.53 \\
\hline \multirow[t]{3}{*}{ Least square estimation } & LSE-1 & 3.75 & 3.44 & 3.24 & 3.36 & 3.20 & 3.13 \\
\hline & LSE-2 & 4.21 & 4.78 & 3.70 & 5.12 & 7.56 & 4.99 \\
\hline & LSE-3 & 3.31 & 3.61 & 3.71 & 2.53 & 3.46 & 3.95 \\
\hline \multirow{2}{*}{ Minimization of APE } & MinAPEi & 5.40 & 6.26 & 5.79 & 6.68 & 10.11 & 8.85 \\
\hline & MinMaxAPEi & 4.94 & 5.40 & 4.48 & 6.95 & 9.03 & 6.73 \\
\hline
\end{tabular}

Bold numbers represent best results in candidate forecasts or combination forecasts for the month

SDA involves finding best correlated days i.e., similar days and forecasting the load as weighted linear combination of the similar days. The weights to combined similar days can be obtained from the historical data by solving a linear set of equations. It uses historical load and temperature and humidity as explanatory variables and produces next day profile.

In ANN model, the network architecture has one input layer, two hidden layer and one output layer. Activation function is taken as sigmoid function at each hidden layer and pure linear function at output layer. Back-propagation algorithm is used as a learning algorithm to train the network. Number of maximum iterations and error convergence are set as stopping criteria.

In ARIMA model, the series is first made stationary by differencing. Order of the time series is determined by minimum Bayesian information criterion (BIC). Coefficients of auto-regression (AR) and moving average (MA) terms are computed using maximum log likelihood.

Methods (individual and combined) used in the study are listed in table 2. We demonstrate the performance of

Table 4. Comparison of MAPE and SSE of candidate forecasts and their combinations for discom-1 from August 2013 to October 2013 after pre-tuning of candidate forecasts.

\begin{tabular}{|c|c|c|c|c|c|c|c|}
\hline \multicolumn{2}{|l|}{ Forecasting methods } & \multicolumn{3}{|c|}{ MAPE $(\%)$} & \multicolumn{3}{|c|}{$\mathrm{SSE}\left(\times 10^{4}\right)$} \\
\hline Category & Model & August & September & October & August & September & October \\
\hline \multirow[t]{3}{*}{ Candidate forecasts } & ANN & 6.41 & 6.15 & 6.26 & 8.93 & 10.21 & 11.57 \\
\hline & SDA & 3.06 & 2.81 & 3.04 & 2.14 & 2.17 & 2.48 \\
\hline & ARIMA & 3.11 & 3.24 & 2.85 & 2.23 & 3.28 & 2.25 \\
\hline \multirow[t]{2}{*}{ Averaging } & Average & 3.30 & 3.37 & 3.32 & 2.43 & 3.12 & 3.02 \\
\hline & Median & 2.98 & 2.92 & 2.87 & 2.05 & 2.42 & 2.21 \\
\hline Outperformance & PoS & 3.07 & 3.05 & 2.97 & 2.17 & 2.61 & 2.38 \\
\hline \multirow[t]{5}{*}{ Minimization of variance } & BG & 2.97 & 2.84 & 2.78 & 2.02 & 2.31 & 2.11 \\
\hline & MinVar & 3.21 & 2.91 & 3.02 & 2.33 & 2.33 & 2.41 \\
\hline & SSE & 2.95 & 2.83 & 2.80 & 2.01 & 2.30 & 2.12 \\
\hline & MinVarC & 3.02 & 2.80 & 2.81 & 2.07 & 2.20 & 2.13 \\
\hline & Eigenvec & 2.94 & 2.76 & 2.73 & 2.01 & 2.23 & 2.04 \\
\hline \multirow[t]{3}{*}{ Least square estimation } & LSE-1 & 3.16 & 2.95 & 3.01 & 2.34 & 2.40 & 2.45 \\
\hline & LSE-2 & 3.12 & 2.84 & 2.98 & 2.25 & 2.26 & 2.36 \\
\hline & LSE-3 & 3.23 & 3.19 & 3.41 & 2.37 & 2.81 & 3.09 \\
\hline \multirow[t]{2}{*}{ Minimization of APE } & MinAPEi & 3.37 & 3.45 & 3.41 & 2.51 & 3.26 & 3.19 \\
\hline & MinMaxAPEi & 3.12 & 3.11 & 3.01 & 2.24 & 2.74 & 2.52 \\
\hline
\end{tabular}

Bold numbers represent best results in candidate forecasts or combination forecasts for the month 


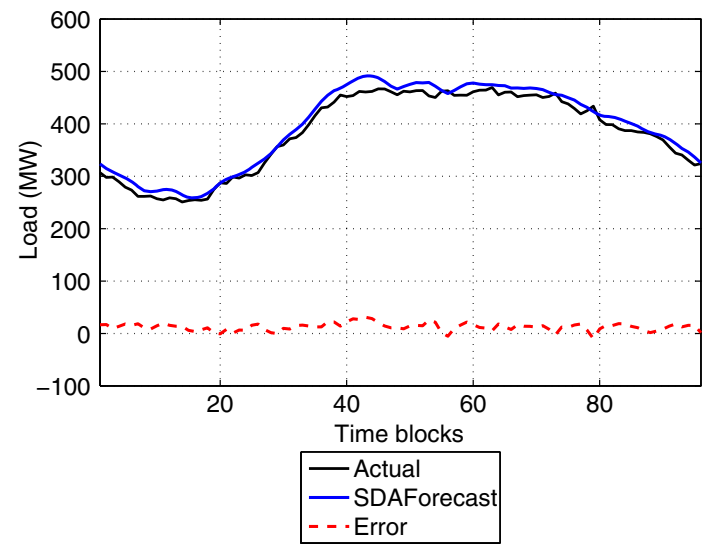

(a)

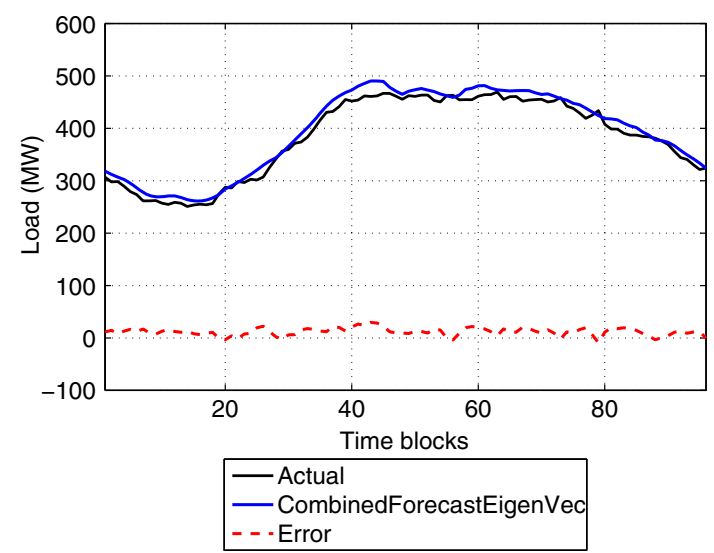

(b)

Figure 2. Plots of actual and forecasted load on August 1, 2013 using a candidate method (SDA) and a combination method (Eigenvec) for discom-1. (a) SDA and (b) eigenvec.

combination approaches on out-of-sample testing. Testing is done for the data from May 2013 to Oct 2013. From recent history, we use previous 15 outcomes of the candidate forecasting models for obtaining weights to combine a-step-ahead candidate forecasts. Anomalous days and holidays are excluded from the case study.

\subsection{Performance of combination approaches for discom-1}

4.1a Combination of sub-optimal forecasts: In this case, we show that if the candidate forecasts are not optimal, combining them produces a forecast with much better accuracy in terms of MAPE or SSE. Comparison of MAPE and SSE for individual and combined forecasts are also shown in table 3. From table 3 , it can be noticed that some combination approaches reduce error up to $30-50 \%$ as compared to candidate forecasts. It is not necessary that the combination always improves accuracy, however, in most cases, it does. In this case, only MinVar combination approach performs poorer than the best among individual forecasts. However, it is evident from performance of MinVarC that adding a constraint of non-negative weights in the minimization of variance problem helps in improving the accuracy. On both the measures of accuracy, discussed here, unconstrained linear regression without a constant (LSE-1) and with a constant (LSE-3) performs better than the other combination approaches.

One advantage of combination of forecasts in this case is that the combined forecast acts as a benchmark. It shows that the candidate forecasts can be improved.

4.1b Combination after pre-tuning of candidate forecasts: In the above case, we have noticed above that there is a scope of optimizing performance of the candidate forecasts. We have tried to improve their performance with changing various parameters and training data size in the candidate forecasting models. From table 4 , it can be noticed that the performance of individual forecasts has now improved. However, ANN does not appear as a good choice for the data set. Results of combination methods show that there is still some improvement in the accuracy as compared to the individual forecasts. This could be due to following reasons.

- All candidate forecasts use same information set, i.e., weather information from a same source. Considering

Table 5. Effect of changing number of historical observations on MAPE (\%) for discom-1 from August 2013 to October 2013.

\begin{tabular}{|c|c|c|c|c|c|c|c|c|c|c|}
\hline \multicolumn{2}{|l|}{ Forecasting methods } & \multicolumn{3}{|c|}{ August } & \multicolumn{3}{|c|}{ September } & \multicolumn{3}{|c|}{ October } \\
\hline Category & Model & 6 Nos. & 10 Nos. & 15 Nos. & 6 Nos. & 10 Nos. & 15 Nos. & 6 Nos. & 10 Nos. & 15 Nos. \\
\hline Outperformance & PoS & 3.23 & 3.13 & 3.07 & 3.17 & 3.10 & 3.05 & 3.02 & 2.98 & 2.97 \\
\hline \multirow[t]{5}{*}{ Minimization of variance } & BG & 3.05 & 2.98 & 2.97 & 2.92 & 2.88 & 2.84 & 2.82 & 2.76 & 2.78 \\
\hline & MinVar & 4.30 & 3.43 & 3.21 & 4.03 & 3.31 & 2.91 & 4.01 & 3.24 & 3.02 \\
\hline & SSE & 3.01 & 2.96 & 2.95 & 2.90 & 2.87 & 2.83 & 2.81 & 2.78 & 2.80 \\
\hline & MinVarC & 3.26 & 3.08 & 3.02 & 3.04 & 2.94 & 2.80 & 2.92 & 2.80 & 2.81 \\
\hline & Eigenvec & 2.95 & 2.90 & 2.94 & 2.85 & 2.80 & 2.76 & 2.81 & 2.73 & 2.73 \\
\hline \multirow[t]{3}{*}{ Least square estimation } & LSE-1 & 5.05 & 3.88 & 3.16 & 4.35 & 3.40 & 2.95 & 4.15 & 3.34 & 3.01 \\
\hline & LSE-2 & 3.92 & 3.35 & 3.12 & 3.68 & 3.18 & 2.84 & 3.80 & 3.22 & 2.98 \\
\hline & LSE-3 & 6.31 & 4.17 & 3.23 & 5.58 & 3.79 & 3.19 & 6.14 & 3.84 & 3.41 \\
\hline \multirow[t]{2}{*}{ Minimization of APE } & MinAPEi & 3.34 & 3.35 & 3.37 & 3.41 & 3.43 & 3.45 & 3.37 & 3.39 & 3.41 \\
\hline & MinMaxAPEi & 3.30 & 3.22 & 3.12 & 3.19 & 3.19 & 3.11 & 2.99 & 2.94 & 3.01 \\
\hline
\end{tabular}


Table 6. Effect of changing number of historical observations on SSE $\left(\times 10^{4}\right)$ for discom-1 from August 2013 to October 2013.

\begin{tabular}{|c|c|c|c|c|c|c|c|c|c|c|}
\hline \multicolumn{2}{|l|}{ Forecasting methods } & \multicolumn{3}{|c|}{ August } & \multicolumn{3}{|c|}{ September } & \multicolumn{3}{|c|}{ October } \\
\hline Category & Model & 6 Nos. & 10 Nos. & 15 Nos. & 6 Nos. & 10 Nos. & 15 Nos. & 6 Nos. & 10 Nos. & 15 Nos. \\
\hline Outperformance & PoS & 2.41 & 2.24 & 2.17 & 2.92 & 2.74 & 2.61 & 2.48 & 2.39 & 2.38 \\
\hline \multirow[t]{5}{*}{ Minimization of variance } & BG & 2.14 & 2.03 & 2.02 & 2.52 & 2.40 & 2.31 & 2.20 & 2.09 & 2.11 \\
\hline & MinVar & 7.31 & 2.66 & 2.33 & 6.73 & 3.27 & 2.33 & 7.49 & 3.27 & 2.41 \\
\hline & SSE & 2.07 & 2.02 & 2.01 & 2.48 & 2.39 & 2.30 & 2.19 & 2.11 & 2.12 \\
\hline & MinVarC & 2.49 & 2.16 & 2.07 & 2.90 & 2.59 & 2.20 & 2.40 & 2.14 & 2.13 \\
\hline & Eigenvec & 2.02 & 1.96 & 2.01 & 2.41 & 2.31 & 2.23 & 2.16 & 2.04 & 2.04 \\
\hline \multirow[t]{3}{*}{ Least square estimation } & LSE-1 & 9.41 & 3.41 & 2.34 & 7.32 & 3.37 & 2.40 & 7.82 & 3.45 & 2.45 \\
\hline & LSE-2 & 3.66 & 2.52 & 2.25 & 5.46 & 3.00 & 2.26 & 6.54 & 3.40 & 2.36 \\
\hline & LSE-3 & 15.44 & 3.93 & 2.37 & 11.95 & 4.07 & 2.81 & 37.83 & 4.74 & 3.09 \\
\hline \multirow[t]{2}{*}{ Minimization of APE } & MinAPEi & 2.48 & 2.50 & 2.51 & 3.18 & 3.23 & 3.26 & 3.12 & 3.16 & 3.19 \\
\hline & MinMaxAPEi & 2.47 & 2.35 & 2.24 & 3.13 & 3.06 & 2.74 & 2.53 & 2.40 & 2.52 \\
\hline
\end{tabular}

different weather information sets may further help in reducing error of load forecast due to erroneous weather forecasts.

- Performance of ANN as compared to two other methods is not good. Combining competitive forecasts may result in good accuracy.

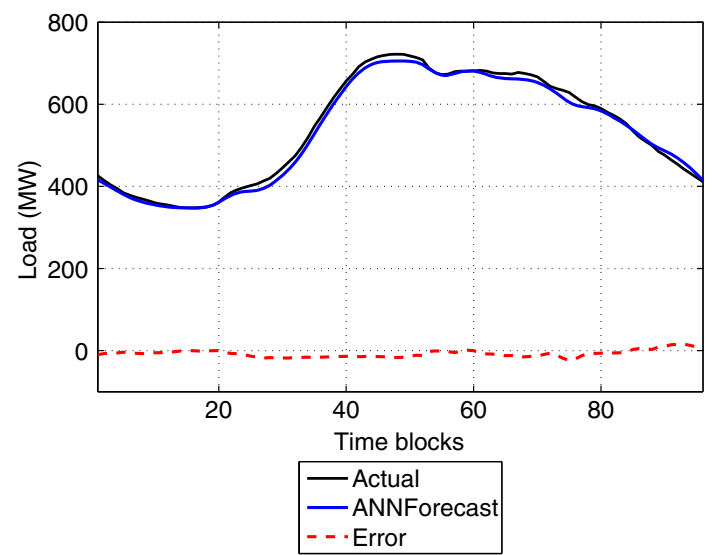

(a)



(b)

Figure 3. Plots of actual and forecasted load on August 1, 2013 using a candidate method (ANN) and a combination method (SSE) for discom-2. (a) ANN and (b) SSE.
Comparing among all the combination approaches, the approach based on eigenvector of error covariance matrix performs better. In the category of averaging methods, median outperforms the simple average method. Minimization of variance based approaches, except MinVar, give combined forecast with better accuracy in terms of MAPE and SSE for most of the cases. By comparing MinVar and MinVarC, it behaves again in similar fashion as in the previous case. Performance of LSE and minimization of APE combination approaches is not good in comparison to the best individual forecaster. Plots for actual load and forecast profiles on August 01, 2013 are shown in figure 2.

4.1c Effect of variation in the number of historical observations: Now, we study the effect of variation in the number of historical observations. We use previously forecasted load values using different forecasting models to estimate optimal weights for combination. Change in the historical window affects the estimation of weights except for the case of averaging. Tables 5 and 6 show that increasing number of historical samples helps in improving the accuracy of combined forecast. Rate of improvement with increase in number of samples is low in the combination approaches except LSE. In LSE, particularly in LSE3 , the rate is very high both in MAPE and SSE.

\subsection{Performance of combination approaches for discom-2}

We, now, do comparative analysis of the combination approaches for another discom in Mumbai. Plots for actual load and forecast profiles on August 01, 2013 are shown in figure 3. The performance of individual and combined forecasts for the period of August 2013 to October 2013 has been shown in table 7 . It can be noticed that forecasting using ANN model gives better result in terms of MAPE among all candidate forecasts. Performance of combination 
Table 7. Comparison of MAPE and SSE of candidate forecasts and their combinations for discom-2 for August 2013 to October 2013.

\begin{tabular}{|c|c|c|c|c|c|c|c|}
\hline \multicolumn{2}{|l|}{ Forecasting methods } & \multicolumn{3}{|c|}{ MAPE $(\%)$} & \multicolumn{3}{|c|}{$\operatorname{SSE}\left(\times 10^{4}\right)$} \\
\hline Category & Model & August & September & October & August & September & October \\
\hline \multirow[t]{3}{*}{ Candidate forecasts } & ANN & 3.11 & 4.10 & 5.46 & 5.44 & 8.75 & 15.80 \\
\hline & SDA & 3.63 & 4.24 & 5.30 & 7.64 & 8.78 & 15.79 \\
\hline & ARIMA & 3.60 & 4.30 & 5.09 & 6.29 & 8.47 & 14.11 \\
\hline \multirow{2}{*}{ Averaging } & Average & 2.90 & 3.68 & 5.00 & 3.81 & 6.17 & 12.19 \\
\hline & Median & 2.69 & 3.62 & 4.73 & 3.26 & 5.93 & 11.23 \\
\hline Outperformance & PoS & 2.70 & 3.64 & 4.84 & 3.30 & 6.10 & 11.79 \\
\hline \multirow[t]{5}{*}{ Minimization of variance } & BG & 2.80 & 3.65 & 5.07 & 3.64 & 6.13 & 12.82 \\
\hline & MinVar & 2.97 & 4.16 & 5.63 & 4.75 & 9.06 & 20.45 \\
\hline & SSE & 2.66 & 3.62 & 4.82 & 3.22 & 5.96 & 11.61 \\
\hline & MinVarC & 2.75 & 3.80 & 5.26 & 3.77 & 6.81 & 14.70 \\
\hline & Eigenvec & 3.13 & 3.81 & 5.13 & 4.79 & 6.69 & 13.42 \\
\hline \multirow[t]{3}{*}{ Least square estimation } & LSE-1 & 3.19 & 4.03 & 5.09 & 4.67 & 7.87 & 12.85 \\
\hline & LSE-2 & 2.74 & 3.84 & 4.82 & 3.63 & 7.15 & 12.03 \\
\hline & LSE-3 & 3.18 & 3.60 & 5.69 & 4.63 & 6.54 & 14.11 \\
\hline \multirow[t]{2}{*}{ Minimization of APE } & MinAPEi & 2.94 & 3.69 & 5.03 & 3.90 & 6.22 & 12.30 \\
\hline & MinMaxAPEi & 2.99 & 3.83 & 4.99 & 4.24 & 7.07 & 13.49 \\
\hline
\end{tabular}

Bold numbers represent best results in candidate forecasts or combination forecasts for the month

using median and SSE gives better forecasts among all approaches.

4.2a Comparison of distribution of forecast errors: de Menezes et al [14] suggested that the combination forecasts should also be evaluated on the basis of distribution of errors. The distribution of errors helps in assessment of risk and uncertainty associated with the forecast. Statistics (e.g., mean, standard deviation, skewness and kurtosis) of individual and combined forecasts are shown in table 8 . It can be noticed that none of the candidate forecasts is unbiased i.e., expected value of error is not close to zero. Combining forecasts reduces the standard deviation and skewness for most of the cases. Skewness tells about the symmetry of distribution around the mean. Reduction in skewness implies improvement in symmetry. Kurtosis gives information about peakedness and longer tail of a distribution. Kurtosis of a normal distribution is three. Excess kurtosis is generally used as a relative measure of kurtosis of a distribution with respect to normal distribution. Excess kurtosis of a distribution is calculated by subtracting three from the kurtosis value of the distribution. In this case, all the distributions have positive excess kurtosis which indicates that the distributions have peakedness and/or longer tails. High peakedness of error distributions of ARIMA and MinAPE can be explained with higher values of excess kurtosis. Positive excess kurtosis may also indicate the presence of outliers.

$4.2 \mathrm{~b}$ Distribution of weights for different combination approaches: For discom-2, we demonstrate the distribution

Table 8. Statistics of individual and combined forecast error for discom-2 from August 2013 to October 2013.

\begin{tabular}{|c|c|c|c|c|c|}
\hline Forecasting methods & & Mean (MW) & Standard deviation (MW) & Skewness & Kurtosis \\
\hline \multirow[t]{3}{*}{ Candidate forecasts } & ANN & 9.94 & 30.73 & -0.15 & 8.60 \\
\hline & SDA & 15.54 & 29.66 & 0.55 & 4.34 \\
\hline & ARIMA & 13.90 & 28.47 & 1.08 & 15.18 \\
\hline \multirow[t]{2}{*}{ Averaging } & Average & 13.13 & 24.48 & -0.02 & 4.88 \\
\hline & Median & 11.39 & 24.10 & 0.03 & 5.12 \\
\hline Outperformance & $\mathrm{PoS}$ & 11.50 & 24.60 & 0.03 & 5.71 \\
\hline \multirow[t]{5}{*}{ Minimization of variance } & BG & 12.98 & 24.85 & 0.10 & 5.57 \\
\hline & MinVar & 13.44 & 31.81 & 1.07 & 9.01 \\
\hline & SSE & 11.63 & 24.24 & 0.02 & 5.73 \\
\hline & MinVarC & 12.52 & 26.90 & 0.36 & 6.26 \\
\hline & Eigenvec & 13.70 & 26.05 & 0.22 & 7.31 \\
\hline \multirow[t]{3}{*}{ Least square estimation } & LSE-1 & 11.95 & 27.19 & -0.12 & 6.54 \\
\hline & LSE-2 & 10.00 & 26.32 & -0.05 & 6.52 \\
\hline & LSE-3 & 10.31 & 27.82 & -0.04 & 5.01 \\
\hline \multirow[t]{2}{*}{ Minimization of APE } & MinAPEi & 13.31 & 24.56 & -0.01 & 4.83 \\
\hline & MinMaxAPEi & 11.66 & 26.96 & 0.55 & 14.51 \\
\hline
\end{tabular}



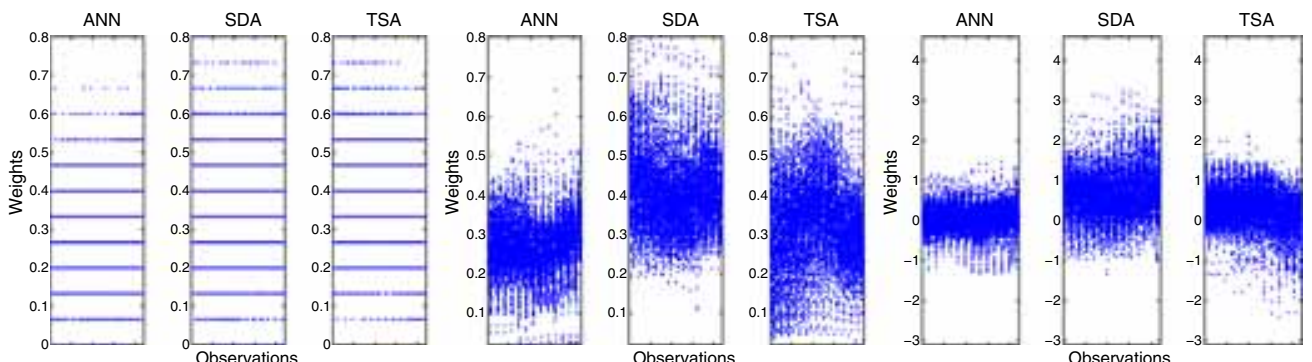

(a)
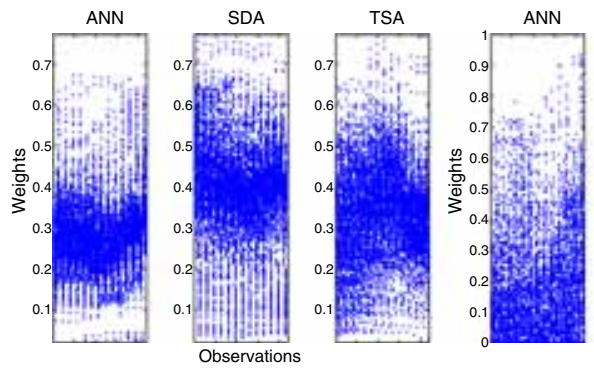

(b)

(c)
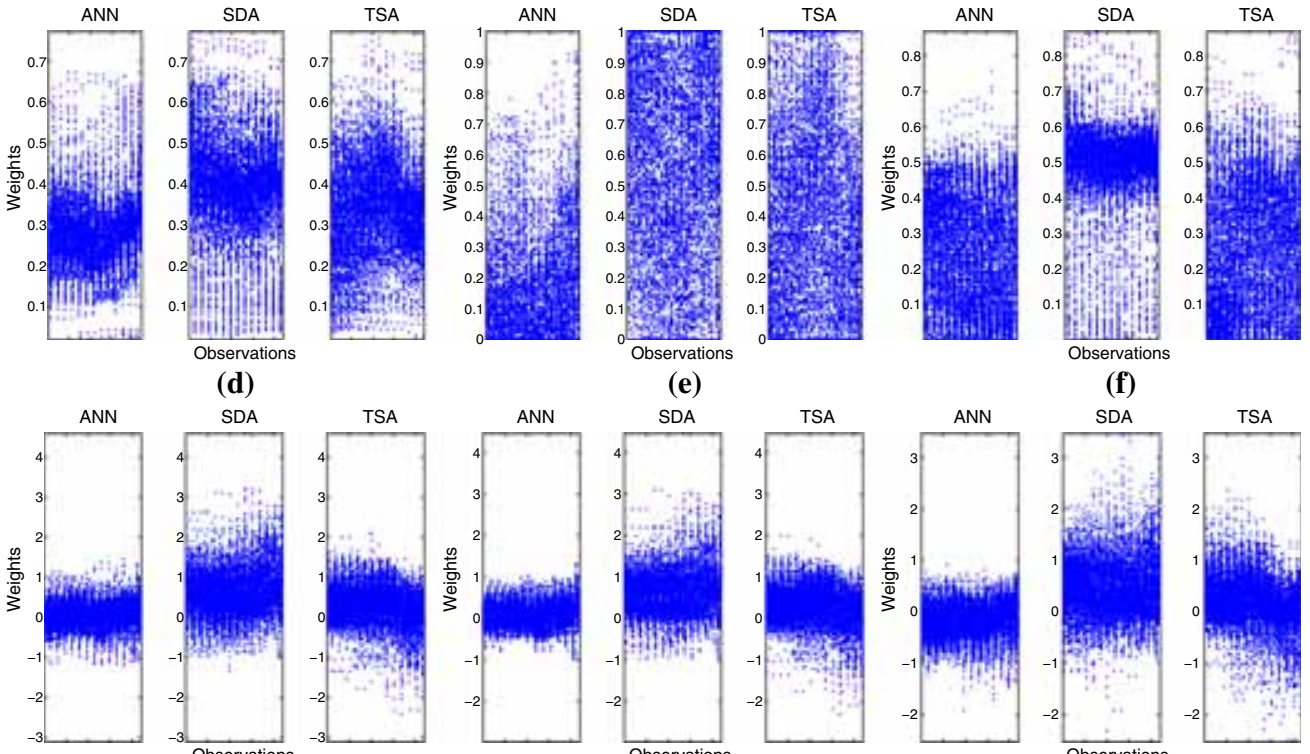

(d)

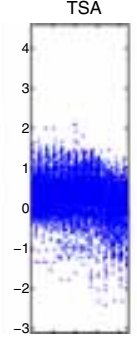

(g)

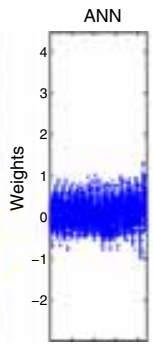

(e)

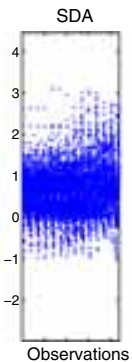

(h)
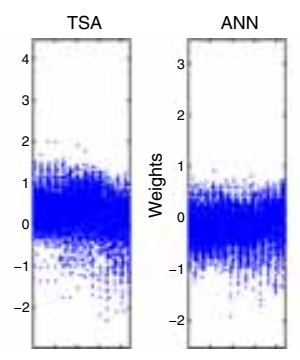

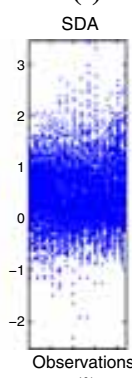

(i)

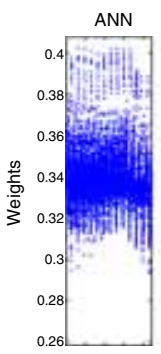

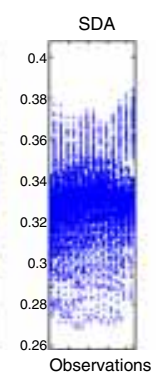

(j)
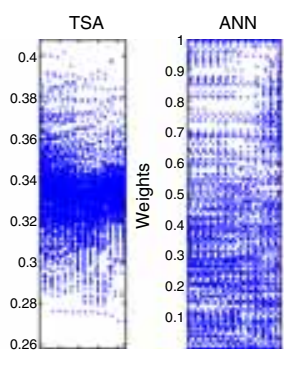

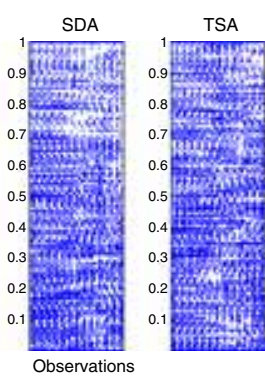

(k)

Figure 4. Distribution of weights for various combination approaches. (a) PoS, (b) BG, (c) MinVar, (d) SSE, (e) MinVarC, (f) Eigenvec, (g) LSE-1, (h) LSE-2, (i) LSE-3, (j) MinAPEi and (k) MinMaxAPEi.

of weights for different combination approaches. Weights, as shown in figure 4, are for all 96 points of all days considered under testing for various categories of combination approaches. Combining forecasts using average method has equal weights for all test instances and hence, has absolute stability of weights. Median approach gives unity weight to the central value of the sorted load forecasts if the number of forecasts are odd. For even number of forecasts the unity weight is divided equally between the two central values of the sorted load forecasts. Weights from averaging combination approaches are not shown here.
Weights in the PoS method will be from the sets of discrete probabilities i.e., $w_{P o S}=\left\{\frac{1}{N}, \frac{2}{N}, \ldots, 1\right\}$. MinVar and the three linear regression approaches viz. LSE-1, LSE2 and LSE-3 can have negative weights. Weights derived using MinAPEi method vary in a comparatively small range and exhibit the better stability of weights compared to other combination approaches. Weights in MinMaxAPEi approach cover the complete span of zero to one and seem to be highly unstable.

It should be noticed that all combination approaches assign non-zero weights to all the candidate forecasts. It 
Table 9. Composite rank (CRank) of discoms on the performance from August 2013 to October 2013.

\begin{tabular}{lrrrrr}
\hline & \multicolumn{2}{c}{ Discom-1 } & & \multicolumn{2}{c}{ Discom-2 } \\
\cline { 2 - 3 } \cline { 5 - 6 } Forecasting models & \multicolumn{1}{c}{ CS } & CRank & & CS & CRank \\
\hline ANN & 4.9649 & 16 & & 1.3006 & 13 \\
SDA & -0.8498 & 1 & & 0.0510 & 12 \\
TSA & 0.3336 & 14 & & 2.4341 & 16 \\
Average & -0.3039 & 12 & & -1.3739 & 2 \\
Median & -0.6640 & 7 & & -1.4999 & 1 \\
PoS & -0.6116 & 9 & & -1.2023 & 5 \\
BG & -0.7795 & 3 & & -1.1164 & 6 \\
MinVar & -0.6218 & 8 & & 1.6893 & 14 \\
SSE & -0.7741 & 4 & & -1.2995 & 4 \\
MinVarC & -0.7914 & 2 & & -0.3872 & 8 \\
Eigenvec & -0.7645 & 5 & & -0.2931 & 10 \\
LSE-1 & -0.4976 & 11 & -0.1587 & 11 \\
LSE-2 & -0.6864 & 6 & & -0.5317 & 7 \\
LSE-3 & -0.2374 & 13 & & -0.3550 & 9 \\
MinAPEi & 2.8678 & 15 & & -1.3517 & 3 \\
MinMaxAPEi & -0.5843 & 10 & & 1.6944 & 15 \\
\hline
\end{tabular}

implies that the candidate forecasts considered are not noncontributory or free riders.

\subsection{Comments on robustness of forecasters}

This empirical analysis shows that combination approaches, in most cases, outperform the individual forecasts in terms of forecast accuracy. Any single combination approach may not guarantee improvement in accuracy for all the data sets. In other words, if correct combination strategy is chosen, then combination approach is likely to be more robust then individual forecasts. Further, analysis of forecast error distribution helps in assessing the risk and uncertainty associated with the forecaster. Skewness and kurtosis give information about the shape of the distribution. Skewness of a forecast error distribution can help a discom in decision making for day-ahead trading or scheduling. For example, in peak hours, when real time power purchase from a market or power exchange is costlier, the discom would prefer a forecaster with positive skewness (more chances of forecast being more than the actual load) for day-ahead scheduling. Further, a discom would also like to minimize chances of outliers in the forecast error distribution. Kurtosis can give indication about the longer tail i.e., the presence of outliers. Consistency in forecast accuracy and absence of outliers lead to a robust forecaster.

We calculate CRank of the two discoms, based on the performance in terms of statistics (i.e., standard deviation and kurtosis) of forecast error distribution and forecast accuracy measure (i.e., MAPE) for the period of August 2013 to October 2013. Weights assigned are $(0.25,0.25$,
0.50 ) respectively. Value of $n$ is taken as 0 . In table 9 , it can be noticed that for discom-1, an individual forecaster SDA ranks first, followed by a combination forecaster MinVarC. Other combined forecasters in the category of minimization of variance also have good rank. For discom-2, average based combined forecasters, in particular median, perform at the top in the CRank. However, the CRank is subjective and demonstrated for a set of weights and power index. A decision maker can calculate the same with the preferred set of weights. For both the discoms, forecasters with CRank equal to one have all the three components of Eq. (10) are as negative. It implies that the top rank forecaster performs better in all performance measures considered.

\section{Conclusions}

Many methods/frameworks have been proposed for STLF. It was observed that no single method outperforms consistently on real life data sets. Therefore, it is advisable that instead of choosing one and discarding remaining methods, a combination approach be tried to derive "best of all". In this paper, this idea has been formalized through notion of robust forecaster which implies consistently good forecast accuracy, lesser shocks and lower standard deviation in the forecast error distribution.

Three individual forecasting methods (SDA, ANN and ARIMA) as well as 13 combination approaches have been evaluated. Then, a ranking scheme based on composite score has been proposed. Based on our empirical analysis, following are the salient observations.

1. When forecasts are suboptimal, combination leads to significant improvements in MAPE and SSE. In such cases, combined forecast can also be used as a benchmark to improve accuracy of individual forecasts. In some sense combination approach incorporates both the idea of hedging and diversification used in the portfolio optimization.

2. Even if the individual forecasts are optimized, the combination approach outperforms the individual forecasts. However, the gains in accuracy are now moderate.

3. Use of larger number of historical observations helps in improving accuracy of combined forecast.

4. To identify a robust forecaster, a composite score (CS) for each forecaster in a given set of forecasting approaches (individual as well as combined) can be calculated. The CS emphasizes on forecast accuracy, spread and length of tail of forecast error distribution. Based on CS, the approaches can be ranked to calculated composite rank (CRank). A forecaster with high CRank can be considered as robust forecaster and can be implemented for decision making in practical scenarios.

5. It is seen that when ranking is done on the superset of individual and combination forecasts, the combination 
forecasts outperform the individual one thereby justifying their need.

\section{References}

[1] Hahn H, Meyer-Nieberg S and Pickl S 2009 Electric load forecasting methods: Tools for decision making. Eur. J. Oper. Res. 199(3): 902-907

[2] Bates J M and Granger C W J 1969 The combination of forecasts. Oper. Res. Q. 20(4):451-468

[3] Wallis K F 2011 Combining forecasts - forty years later. Appl. Finan. Econom. 21: 33-41

[4] Bichpuriya Y, Rao M S S and Soman S 2010 Combination approaches for short term load forecasting. In: IPEC, 2010 Conference Proceedings, pp 818-823

[5] Bordignon S, Bunn D W, Lisi F and Nan F 2013 Combining day-ahead forecasts for British electricity prices. Energy Econom. 35: 88-103

[6] Shen S, Li G and Song H 2011 Combination forecasts of international tourism demand. Ann. Tourism Res. 38(1): 72-89

[7] Newbold P and Granger C W J 1974 Experience with forecasting univariate time series and the combination of forecasts. J. Royal Stat. Soc. S A (General) 137(2): 131-165
[8] Granger C W J and Ramanathan R 1984 Improved methods of combining forecasts. J. Forecast. 3(2):197-204

[9] Lam K, Mui H and Yuen H 2001 A note on minimizing absolute percentage error in combined forecasts. Comput. Oper. Res. 28(11): 1141-1147

[10] Kiartzis S, Kehagias A, Bakirtzis A and Petridis V 1997 Short term load forecasting using a Bayesian combination method. Int. J. Electr. Power Energy Syst. 19(3): 171-177

[11] Barghinia S, Kamankesh S, Mahdavi N, Vahabie A H and Gorji A 2008 A combination method for short term load forecasting used in Iran electricity market by NeuroFuzzy, Bayesian and finding similar days methods. In: 5th International Conference on European, Electricity Market, 2008. EEM 2008. pp 1-6

[12] Fan S, Chen L and Lee W-J 2009 Short-term load forecasting using comprehensive combination based on multimeteorological information. IEEE Trans. Ind. Appl. 45(4): 1460-1466

[13] DeCarlo L T 1997 On the meaning and use of kurtosis. Psychol. Methods 2(3): 292-307

[14] de Menezes L M, Bunn D W and Taylor J W 2000 Review of guidelines for the use of combined forecasts. Eur. J. Oper. Res. 120(1): 190-204 